\title{
Intrinsic resonance representation of quantum mechanics
}

\section{Carioli, M.; Heller, E.J.; Møller, Klaus Braagaard}

Published in:

Journal of Chemical Physics

Link to article, DOI:

10.1063/1.473911

Publication date:

1997

Document Version

Publisher's PDF, also known as Version of record

Link back to DTU Orbit

Citation (APA):

Carioli, M., Heller, E. J., \& Møller, K. B. (1997). Intrinsic resonance representation of quantum mechanics. Journal of Chemical Physics, 106(20), 8564-8571. https://doi.org/10.1063/1.473911

\section{General rights}

Copyright and moral rights for the publications made accessible in the public portal are retained by the authors and/or other copyright owners and it is a condition of accessing publications that users recognise and abide by the legal requirements associated with these rights.

- Users may download and print one copy of any publication from the public portal for the purpose of private study or research.

- You may not further distribute the material or use it for any profit-making activity or commercial gain

- You may freely distribute the URL identifying the publication in the public portal

If you believe that this document breaches copyright please contact us providing details, and we will remove access to the work immediately and investigate your claim. 


\title{
Intrinsic resonance representation of quantum mechanics
}

\author{
M. Carioli and E. J. Heller \\ Department of Physics, Harvard University and Harvard-Smithsonian Center for Astrophysics, \\ Cambridge, Massachusetts 02138 \\ K. B. Møller \\ Department of Physics, Harvard University, Cambridge, Massachusetts 02138, \\ and Department of Chemistry, Technical University of Denmark, DTU-207, DK-2800 Lyngby, Denmark
}

(Received 14 January 1997; accepted 20 February 1997)

\begin{abstract}
The choice of basis states in quantum calculations can be influenced by several requirements, and sometimes a very natural basis suggests itself. However often one retreats to a "merely complete" basis, whose coefficients in the eigenstates carry little physical insight. We suggest here an optimal representation, based purely on classical mechanics. "Hidden" constants of the motion and good actions already known to the classical mechanics are thus incorporated into the basis, leaving the quantum effects to be isolated and included by small matrix diagonalizations. This simplifies the hierarchical structure of couplings between "zero-order" states. We present a (non-perturbative) method to obtain such a basis-state as solutions to a certain resonant Hamilton-Jacobi equation.
\end{abstract}

(C) 1997 American Institute of Physics. [S0021-9606(97)02020-5]

\section{INTRODUCTION}

There has been a revived interest recently in semiclassical theories based on the Fourier analysis of classical quasiperiodic motion. ${ }^{1}$ These theories were developed in the seventies and eighties by several authors as methods to implement Einstein-Brillouin-Keller (EBK) quantization of non-separable multidimensional systems ${ }^{2-6}$ (a recent review by Child ${ }^{7}$ contains an extensive list of references to the numerous works in this field). Non-separable multidimensional systems are the objects of many studies and applications in various fields, from nuclear physics to astronomy. In molecular physics, the correspondence between the classical and quantum mechanics of systems of non-linear coupled oscillators is of paramount importance for the understanding and fitting molecular of spectra.

In its original form the EBK quantization scheme requires that the classical motion is regular, i.e., that the motion is restricted to invariant tori in phase space, although some empirical evidence that a tori quantization scheme could yield surprisingly good results even when tori do not exist classically was found by Swimm and Delos ${ }^{8}$ in the late seventies and later confirmed by Reinhardt et al. ${ }^{9}$ As suggested by the KAM theorem the classical dynamics of a coupled oscillator system is dominated by regular motion at low energies which implies that almost everywhere in phase space it is possible to find $N$ constants of the motion ("good actions"). If the system is non-resonant (the frequencies are incommensurate) the good actions are global in phase space and the system is separable. In other words, the HamiltonJacobi equation has one solution valid for all phase space.

If the system exhibits resonances it is no longer completely separable. On the other hand, if the motion is still predominantly regular at low energies $N$ good actions can be found almost everywhere in phase space. The actions are only local; different good actions exist in different regions of phase space. A well-known example of this is the separation of the dynamics of two identical interacting oscillators into "local" modes characterized by one set of good actions and "normal" modes characterized by another set of good actions induced by a 1:1 resonance (see, e.g., Ref. 10). Thus, in a resonant, but near integrable, system the HamiltonJacobi equation has many solutions valid in different regions of phase space.

From a semi-classical point of view, the existence of only locally good actions poses a problem. Each of these actions may be quantized by imposing the EBK conditions, but this leaves the distinct regions of phase space decoupled. In systems with some symmetry, or with a high density of states, the decoupling may result in degeneracies or near degeneracies in the obtained EBK energies where the exact quantum energies are non-degenerate. This phenomenon is known as dynamical tunneling. ${ }^{11,12}$ Recently, it has been suggested that the EBK energies should be refined by introducing the tunneling through complex solutions of the Hamilton-Jacobi equation. ${ }^{13}$

Another approach is to remain in a global action-angle description of the classical Hamiltonian and quantize using a uniform approximation scheme, either of the Schödinger type where the action variables are replaced by differential operators $^{14,15}$ or of the Heisenberg type where the Hamiltonian is represented by a matrix. ${ }^{16-19}$ Essentially, the problem is approximated by a system with a single resonance, obtained for example by ignoring, or averaging over other resonances, treating them as fast variables. The resonance is then quantized exactly (i.e., "uniformly") rather than semiclassically. In this way the tunnel splitting are found correctly. The simplicity of these schemes inherently depend on the simplicity of the action-angle representation of the classical Hamiltonian, that is, its angular dependence. ${ }^{9,14,15,18,19}$ Prior to quantization, it is therefore desirable to simplify the classical Hamiltonian by canonically transforming the original coordinates (the action-angle variables of the uncoupled 
system) to a set of "optimal" action-angle variables that reduce the angular dependence as much as possible. This transformation can (to a given order) be found via resonant classical perturbation theory ${ }^{20}$ resulting in a normal form approximation to the Hamiltonian. To lowest order, the Hamiltonian obtained this way equals the Hamiltonian in the original coordinates where all angular terms not corresponding to resonances between the uncoupled oscillators have been omitted. This simple approach was applied by Kellman and Lynch $^{17}$ in a Heisenberg matrix context and they obtained good results at low energy for two kinetically coupled Morse oscillators.

For a system of coupled harmonic oscillators, higher order normal forms can be found using the BirkhoffGustavson method. ${ }^{21,8,22}$ In its original form, the BirkhoffGustavson method takes into account only the resonances due to exact commensurabilities between the frequencies of the harmonic oscillators and will therefore diverge for nearresonant systems. To overcome this divergence several authors have set up methods that treat resonances and nearresonances on equal footing. ${ }^{15,18,19}$

The idea behind all these perturbation treatments is to average over "fast terms.", These may, however, become important as the energy is increased ${ }^{17}$ and any perturbation method will break down.

In this paper we suggest an alternative, non-perturbative, route to a set of global action-angle variables that simplifies the Hamiltonian. This is a self-consistent approach that treats resonances, near-resonances and fast terms on an equal footing. The idea is similar to the idea behind the HamiltonJacobi equation: A set of trial action-angle variables (e.g., the good actions of the uncoupled oscillators and their conjugate angles) are written in terms of a new set of yet unknown action-angle variables. The Hamilton-Jacobi equation is obtained requiring that the Hamiltonian be independent of the new angles. For non-separable systems this requirement is clearly too restrictive to allow for a global solution, since intervening resonance zones locally destroy any fixed set of good actions. We therefore suggest an iterative approach where a "trial form" of the Hamiltonian is gradually allowed to contain more and more combinations of the new angles until a global solution of the modified Hamilton-Jacobi equation can be found. Since we do not insist on the absence of angle variables the resulting Hamiltonian can contain isolated resonance zones. To find a global solution of the desired accuracy (when it exists) we implement an iterative scheme similar to the Chapman-GarrettMiller (CGM) method developed for solving the HamiltonJacobi equation in Fourier space. ${ }^{3}$

In a resonant system of coupled harmonic oscillators at sufficiently low energy where the primary resonances dominate the dynamics, the iterative procedure presented here is expected to converge for a trial Hamiltonian including resonant combinations corresponding to the primary resonances. In this case the iterative method thus provides an alternative route to a resonant normal form to the perturbative BirkhoffGustavson approach. Some formal considerations toward this direction were made previously, e.g., Greenberg et al. at the end of their paper. ${ }^{1}$ The usage of the CGM method to obtain a resonant Hamiltonian when the system is slightly nonresonant has also been suggested by Farrelly and Uzer. ${ }^{15}$ Farrelly and Uzer, however, used the CGM in its original form and did the iteration only once, followed by a perturbative resonance analysis, whereas we suggest to carry out the iteration to self-consistency in the trial Hamiltonian.

Since our classical analysis is performed in Fourier space, the Hamiltonian is most conveniently quantized implementing the Heisenberg type uniform approximation of Jaffé et al. ${ }^{16}$

\section{SEMI-CLASSICAL REPRESENTATION OF THE HAMILTONIAN}

The failure of the EBK quantization to reproduce splittings in resonant or nearly resonant systems is in some sense due to an inconsistent representation of the Hamiltonian in terms of a basis. A semi-classical basis is given by a set of (global) action-angle variables $(\mathbf{I}, \boldsymbol{\phi})$ which are related to physical coordinates $\mathbf{p}$ and $\mathbf{q}$ through a canonical transformation. The action variables I can be thought of as good actions for some separable Hamiltonian $H_{s}$. In a uniform version of EBK quantization the EBK quantized tori of $H_{s}$ play the role of a semi-classical basis in which the nonseparable Hamiltonian of interest, $H$, is diagonalized. Jaffé et $a l .{ }^{16}$ have introduced such a uniform approach derived from the classical theory of the Wigner density for separable systems ${ }^{16}$ and more recently it has been related to a quantum variational principle. ${ }^{1}$ This approach yields a semi-classical version of Heisenberg's matrix mechanics where the diagonal elements are the mean values of $H$ on the EBK tori of $H_{s}$ and the off diagonal coupling elements are higher order Fourier components of $H$ evaluated on the invariant torus of $H_{s}$ intermediate to the two EBK tori that the matrix element couples.

The n'th EBK torus of the separable Hamiltonian $H_{s}$ is defined through the condition $\mathbf{I}_{n}=\hbar(\mathbf{n}+\mathbf{1} / 2)$; and the semiclassical representation of $H$ is then ${ }^{16}$

$$
\begin{aligned}
\langle\mathbf{n}|H| \mathbf{m}\rangle & =\left.(2 \pi)^{-N} \int d \boldsymbol{\phi} H(\mathbf{I}, \boldsymbol{\phi}) e^{-i(n-m) \boldsymbol{\phi}}\right|_{I=\bar{I}_{n+m}} \\
& =H_{n-m}\left(\overline{\mathbf{I}}_{n+m}\right),
\end{aligned}
$$

where $\overline{\mathbf{I}}_{n+m}=\left(\mathbf{I}_{n}+\mathbf{I}_{m}\right) / 2$. Thus the element coupling the n'th and the m'th EBK tori of $H_{s}$ is the $\mathbf{n}-\mathbf{m}$ 'th Fourier component of $H$ evaluated on the intermediate invariant torus of $H_{s}$. This approach obviously leads to a diagonal matrix containing the EBK energies for a separable $H$ if one uses the invariant tori of $H$ as a basis. In fact, we see that $H$ does not need to be truly separable in order to use invariant tori of $H$ as a basis; it is sufficient that $H$ possesses invariant tori of the same family at half-integer actions. The matrix will then contain the EBK energies in the diagonal but there might be non-vanishing off diagonal elements. In other words, in this scheme narrow resonance zones or stochastic regions can be handled; their effect shows up in the off diagonal elements in Eq. (1) and the dynamical tunnel splittings they induce are correctly accounted for. 
However a quasi-integrable system with larger resonance zones, large enough to support EBK quantized tori, requires modified approaches. The simple EBK energies corresponds to energies obtained using the uniform method with different bases in different regions in phase space, i.e., local bases, and splittings are therefore not accounted for correctly.

In order to get a simple coupling structure of the semiclassical matrix a proper choice of basis (EBK tori and their intermediates of some $H_{s}$ ) is crucial. Since the basis states are coupled by the Fourier components of $H$, it is desirable to find a set $(\mathbf{I}, \boldsymbol{\phi})$ in which $H$ has a simple dependence on the angles $\boldsymbol{\phi}$. This is a purely classical question and thus calls for a classical analysis. Let us therefore recapitulate the elements of a classical resonance analysis ${ }^{20}$ and study the importance of the various angle-dependent terms. We consider a Hamiltonian of the form $H_{0}+\epsilon V$ where $H_{0}$ is a system of uncoupled oscillators and $\epsilon V$ is a small coupling term. Taking $(\mathbf{J}, \boldsymbol{\theta})$ to be the basis suggested by the separable $H_{0}$ the Hamiltonian may be written as

$$
H(\mathbf{J}, \boldsymbol{\theta})=H_{0}(\mathbf{J})+\epsilon \sum_{n \neq 0} \sum_{p \geqslant 0} V_{p n}(\mathbf{J}) e^{i p n \boldsymbol{\phi}},
$$

where $\mathbf{n}$ is a vector of integers with no common factors (indicated by the prime on the summation sign). The terms in the sum with an $\mathbf{n}$ containing both positive and negative numbers represent non-linear resonances. Whether these resonances are "active" (giving rise to resonance zones) can to some extent be determined from the commensurability of the zeroth-order frequencies $\omega_{i}(\mathbf{J})=\partial H_{0}(\mathbf{J}) / \partial J_{i}$ in the sense that only terms for which a resonance condition,

$$
\mathbf{n} \cdot \boldsymbol{\omega}(\mathbf{J}) \approx 0,
$$

is met give rise to resonance zones. In the following we use the symbol $\mathbf{r}$ to denote vectors satisfying such a condition.

Dynamically speaking these terms are "slow" whereas the terms not satisfying such relations which includes the terms with $\mathbf{n}$ having elements all of the same sign are "fast." To first order classical perturbation theory tells us that the Hamiltonian in Eq. (2) can be approximated by an effective Hamiltonian obtained by averaging out the fast terms leaving only Fourier components with vectors satisfying Eq. (3). ${ }^{20}$ This, in effect, corresponds to a first order change of basis. ${ }^{14}$ Implementing higher order perturbation theory, the basis can be improved to get a better approximate Hamiltonian that still contains only Fourier terms that originate from active resonances.

The number of active resonances usually depends on the total energy, increasing when the energy is increased. If the number of active resonances is smaller than the number of degrees of freedom the change of basis may reveal (approximate) dynamical constants which play the role of good quantum numbers in a semi-classical theory. These constants of the motion are combinations of basis actions $K=\mathbf{q} \cdot \mathbf{I}^{\prime}$ where the vectors $\mathbf{q}$ span a space orthogonal to the space spanned by the resonance $\mathbf{r}$ vectors ${ }^{23}$ giving rise to the quantum numbers $k=\mathbf{q} \cdot(\mathbf{n}+\mathbf{1} / 2)$.
This is readily deduced from Eq. (1). Let us for simplicity consider a two dimensional system which possesses only an $r: s$ resonance coupling. Then the Hamiltonian can be written as

$$
H(\mathbf{I}, \boldsymbol{\phi})=\sum_{p} H_{p(r,-s)}(\mathbf{I}) e^{i p\left(r \phi_{1}-s \phi_{2}\right)} .
$$

$K=s I_{1}+r I_{2}$ is a constant of the motion for the classical system. ${ }^{20}$ From Eq. (1) it follows that the semi-classical Hamiltonian can be written as

$$
\langle\mathbf{n}|H| \mathbf{m}\rangle=H_{p(r,-s)}\left(\overline{\mathbf{I}}_{n+m}\right) \delta_{n_{1}, m_{1}+p r} \delta_{n_{2}, m_{2}-p s},
$$

which is a block diagonal matrix each block characterized by a fixed value of $k=s\left(n_{1}+1 / 2\right)+r\left(n_{2}+1 / 2\right)$. In other words, states with different values of $k$ do not mix. Even when the number of active resonances equals or exceeds the number of degrees of freedom the basis found by perturbation theory simplifies the semi-classical matrix. ${ }^{17-19}$

Although there is empirical evidence that the approximate Hamiltonian obtained by perturbation theory may give good quantum results even when the classical dynamics of the approximate Hamiltonian differs significantly from the dynamics of the real Hamiltonian ${ }^{9,24,18}$ it is an inherent problem of the perturbative approach that one does not know $a$ priori how well the resulting Hamiltonian approximates the real Hamiltonian. Furthermore, as pointed out by Lynch and Kellman $^{17}$ at higher energy the terms that are considered to be fast may become important. These are, formally, not present in a Hamiltonian obtained by perturbation theory and must, so to speak, be put in by hand on top of the perturbative result.

In the present work we therefore suggest a nonperturbative way to find an optimal basis. For nearly separable systems Chapman, Garrett and Miller (CGM) ${ }^{3,25}$ have developed a non-perturbative method for finding EBK invariant tori. Using a generalized version of this method we search for a family of tori with integer or half-integer actions (which then become the basis) where the non-separable Hamiltonian can be represented by as few n's as possible. Since the basis constructed this way takes into account the actual resonance structure of the system, a representation in such a basis is termed an Intrinsic Resonance Representation (IRR). First, we summarize the original CGM method.

\section{INVARIANT TORI}

We briefly review the CGM method to find invariant tori. This method essentially solves iteratively the HamiltonJacobi equation in order to determine the good actions. Assume that the Hamiltonian is expressed in a zeroth order set of action-angle variables $(\mathbf{J}, \boldsymbol{\theta})$ where $\boldsymbol{\theta}$ is non-cyclic. The goal is then to determine a canonical transformation $(\mathbf{J}, \boldsymbol{\theta}) \rightarrow(\mathbf{I}, \boldsymbol{\phi})$ such that the Hamiltonian depends on the actions I only. The Hamilton-Jacobi characteristic equation can only be solved provided that an invariant torus with action integrals I exists (which is quite possible by virtue of the KAM theorem). To solve the Hamilton-Jacobi equation using an iterative scheme, the actions $\mathbf{J}$ should define a torus 
that is "topologically close" to the target torus (the transformation from $\mathbf{J}$ to $\mathbf{I}$ must be a continuous deformation of the identity). It is therefore clear that when phase space is divided into topologically distinct zones, different zeroth order set action-angle variables must be utilized to obtain invariant tori in their respective regions of phase space in the CGM approach.

The generating function for the transformation $S(\mathbf{I}, \boldsymbol{\theta})$ is therefore written as $S(\mathbf{I}, \boldsymbol{\theta})=\mathbf{I} \cdot \boldsymbol{\theta}+G(\mathbf{I}, \boldsymbol{\theta})$ such that

$$
\begin{aligned}
& \mathbf{J}=\mathbf{I}+\nabla G(\mathbf{I}, \boldsymbol{\theta}), \\
& \boldsymbol{\phi}=\boldsymbol{\theta}+\nabla_{I} G(\mathbf{I}, \boldsymbol{\theta}),
\end{aligned}
$$

and the Hamilton-Jacobi equation then reads as

$$
H(\mathbf{I}+\nabla G, \boldsymbol{\theta})=E(\mathbf{I}) .
$$

The CGM method solves this equation in Fourier space in which the generating function is represented as

$$
G(\mathbf{I}, \boldsymbol{\theta})=\sum_{k}^{\prime} G_{k}(\mathbf{I}) e^{i k \boldsymbol{\theta}},
$$

where the prime on the sum implies that the constant term $\mathbf{k}=\mathbf{0}$ is omitted. Defining $H^{\prime}(\mathbf{I}, \boldsymbol{\theta}) \equiv H(\mathbf{J}(\mathbf{I}, \boldsymbol{\theta}), \boldsymbol{\theta})$ we can write

$$
H^{\prime}(\mathbf{I}, \boldsymbol{\theta})=\sum_{n} H_{n}^{\prime}\left[\left\{G_{k}\right\}\right](\mathbf{I}) e^{i n \boldsymbol{\theta}},
$$

where

$$
\begin{aligned}
H_{n}^{\prime}\left[\left\{G_{k}\right\}\right](\mathbf{I})= & (2 \pi)^{-N} \int d \boldsymbol{\theta} e^{-i n \boldsymbol{\theta}} H(\mathbf{I} \\
& \left.+\sum_{k}{ }^{\prime} i \mathbf{k} G_{k}(\mathbf{I}) e^{i k \boldsymbol{\theta}}, \boldsymbol{\theta}\right)
\end{aligned}
$$

and the equations to solve become the non-linear functional equations,

$$
H_{n}^{\prime}\left[\left\{G_{k}\right\}\right](\mathbf{I})=0, \quad \mathbf{n} \neq \mathbf{0} .
$$

For a $G$ that solves these equations, the energy on the invariant torus determined by the value of $\mathbf{I}$ is given by $E(\mathbf{I})=H_{0}^{\prime}\left[\left\{G_{k}\right\}\right](\mathbf{I})$.

A study of the convergence properties of iteration schemes to solve Eq. (12) has been made by Warnock and Ruth. ${ }^{25}$ Kaasalainen and Binney have devised a scheme to construct suitable actions $\mathbf{J}$ under rather general conditions. ${ }^{26}$ Implementing a Newton-Raphson iteration scheme, at each step of the iteration one solves the following linear system $(\mathbf{n} \neq \mathbf{0})$ :

$$
\sum_{k^{\prime}}{ }^{\prime} \frac{\partial H_{n}^{\prime}\left[\left\{G_{k}^{(n-1)}\right\}\right]}{\partial G_{k^{\prime}}} \delta G_{k^{\prime}}^{(n)}=H_{n}^{\prime}\left[\left\{G_{k}^{(n-1)}\right\}\right],
$$

where $G_{k}^{(n-1)}$ denotes the Fourier transform of $G$ at the $(n-1)$ 'th iteration and $G_{k}^{(n)}=G_{k}^{(n-1)}+\delta G_{k}^{(n)}$.

The first iteration is obtained by setting all $G_{k}^{(0)}=0$, so that $H_{n}^{\prime(0)}$ is the Fourier transform of the Hamiltonian on the torus defined by zeroth-order $\mathbf{J}$. During the iteration $\mathbf{I}$ is just a fixed parameter which is set to the values desired for the actions of the invariant torus. Thus, at each step the action map, $\mathbf{J}(\mathbf{I}, \boldsymbol{\theta})=\mathbf{I}+\nabla G^{(n)}(\mathbf{I}, \boldsymbol{\theta})$, defines a new torus with the same action integrals $\oint_{\epsilon} \mathbf{p} \cdot d \mathbf{q}=\mathbf{I}_{\epsilon}, \epsilon=1, \ldots, N$. It is on this new torus that all Fourier transforms are evaluated. The sequence of generating functions $G^{(n)}(\mathbf{I}, \boldsymbol{\theta})$ is supposed to converge to the solution $G(\mathbf{I}, \boldsymbol{\theta})$ of Eq. (8). The iteration is stopped when the action map defines a torus where the Hamiltonian is constant. It is the fact that $\mathbf{I}$ is a constant parameter set from the beginning that makes this method suitable for EBK quantization.

The original CGM method never needs the angle map, $\boldsymbol{\phi}=\boldsymbol{\theta}+\nabla_{I} G(\mathbf{I}, \boldsymbol{\theta})$. However, for what follows we need an approach where both the new action and angles are known. To do this, at each step of the iteration we find the angles conjugate to the actions obtained at that step. The Fourier transforms of the Hamiltonian on the torus are evaluated in terms of the new angles; i.e., the coordinate system is changed at each step. The Hamiltonian is represented in a set of canonically conjugate coordinates during the process of iteration, which is desirable in the next section where the Hamiltonian is allowed some dependence of the final angles. The new angles at step $n$ is given by

$$
\boldsymbol{\phi}^{(n)}=\boldsymbol{\theta}+\nabla_{I} G^{(n)}(\mathbf{I}, \boldsymbol{\theta}),
$$

which can be inverted to obtain $\boldsymbol{\theta}=\boldsymbol{\theta}\left(\mathbf{I}, \boldsymbol{\phi}^{(n)}\right)$ using a scheme provided by Warnock and Ruth. ${ }^{25}$

\section{CONSTRUCTION OF AN OPTIMAL BASIS}

As described in Sec. II, an optimal semi-classical basis is a single family of tori on which the Hamiltonian under consideration has a simple angular dependence. This basis consists of the usual EBK tori of the Hamiltonian only if it is nearly separable. In this section we suggest a generalization of the CGM method which can be used to find such a family of tori in the non-separable case and which, at the same time, determines the Fourier components of the Hamiltonian on these tori.

First, let us consider a coupled system containing a single primary resonance with the resonance vector $\mathbf{r}$. Instead of seeking action-angle variables $(\mathbf{I}, \boldsymbol{\phi})$ where the Hamiltonian depends only on the action integrals I we seek a set of action-angle variables where the Hamiltonian is on a resonant form, that is, a transformation such that $H(\mathbf{J}, \boldsymbol{\theta})=\widetilde{H}(\mathbf{I}, \mathbf{r} \cdot \boldsymbol{\phi})$ where $\mathbf{r}$ is a resonance vector. In order to do this, we search for a generating function,

$$
G(\mathbf{I}, \boldsymbol{\theta})=\sum_{k}{ }^{\prime \prime} G_{k}(\mathbf{I}) e^{i k \boldsymbol{\theta}},
$$

where the double prime on the sum implies that the terms $\mathbf{k}=p \mathbf{r}$ are omitted; that satisfies a modified Hamilton-Jacobi equation:

$$
H(\mathbf{I}+\nabla G, \boldsymbol{\theta})=\widetilde{H}(\mathbf{I}, \mathbf{r} \cdot \boldsymbol{\phi}) .
$$

Defining $H^{\prime \prime}(\mathbf{I}, \boldsymbol{\phi}) \equiv H(\mathbf{J}(\mathbf{I}, \boldsymbol{\phi}), \boldsymbol{\theta}(\mathbf{I}, \boldsymbol{\phi}))$ we can write the Hamiltonian as a Fourier series in the new angles, 


$$
H^{\prime \prime}(\mathbf{I}, \boldsymbol{\phi})=\sum_{n} H_{n}^{\prime \prime}\left[\left\{G_{k}\right\}\right](\mathbf{I}) e^{i n \boldsymbol{\phi}},
$$

with the Fourier components being

$$
\begin{aligned}
H_{n}^{\prime \prime}\left[\left\{G_{k}\right\}\right](\mathbf{I}) & \\
& =(2 \pi)^{-N} \int d \boldsymbol{\phi} e^{-i n \boldsymbol{\phi}} H\left(\mathbf{I}+\sum_{k}{ }^{\prime} i \mathbf{k} G_{k}(\mathbf{I}) e^{i k \boldsymbol{\phi},}, \boldsymbol{\theta}\right),
\end{aligned}
$$

where the old angles are functions of the new action-angle variables, $\boldsymbol{\theta}=\boldsymbol{\theta}(\mathbf{I}, \boldsymbol{\phi})$. The equations to solve then become the non-linear functional equations,

$$
H_{n}^{\prime \prime}\left[\left\{G_{k}\right\}\right](\mathbf{I})=0, \quad \mathbf{n} \neq p \mathbf{r}
$$

Again implementing a Newton-Raphson iteration scheme, at each step of the iteration one solves the following linear system $(\mathbf{n} \neq p \mathbf{r})$ :

$$
\sum_{k^{\prime}}{ }^{\prime \prime} \frac{\partial H_{n}^{\prime \prime}\left[\left\{G_{k}^{(n-1)}\right\}\right]}{\partial G_{k^{\prime}}} \delta G_{k^{\prime}}^{(n)}=H_{n}^{\prime \prime}\left[\left\{G_{k}^{(n-1)}\right\}\right],
$$

followed by the inversion of Eq. (14) to obtain $\boldsymbol{\theta}=\boldsymbol{\theta}\left(\mathbf{I}, \boldsymbol{\phi}^{(n)}\right)$. At the beginning of the iteration we set $G_{k}^{(0)}=0$ implying that $\boldsymbol{\phi}^{(0)}=\boldsymbol{\theta}$. The iteration is stopped when the Fourier components $H_{n}^{\prime \prime}\left[\left\{G_{k}\right\}\right](\mathbf{I}), \mathbf{n} \neq p \mathbf{r}$ are smaller than the error one decides to tolerate. The desired resonant Hamiltonian is then given by

$$
\widetilde{H}(\mathbf{I}, \boldsymbol{\phi})=\sum_{p} H_{p r}^{\prime \prime}(\mathbf{I}) e^{i p r \boldsymbol{\phi}} .
$$

At this point a few things about convergence of the method should be noted. If the Hamiltonian is not truly single resonant, the above expression cannot be obtained exactly. However, if the stochastic regions and the resonance zones due to higher order resonances are very narrow, the procedure is expected to converge in almost all phase space. In this connection it should be kept in mind that the semi-classical representation only requires a discrete set of tori, namely those having integer and half-integer actions.

Finally, it should be recognized that the phase-space topology of resonant and nearly resonant systems are alike, and the resonant vector must be included in both cases to obtain convergence. In this way the method does not distinguish between these two cases.

Above, it was assumed that only one resonance is active. If more resonances or fast terms are active, these have to be included in order to make the iteration converge. As in the original CGM method, the family of initial tori must be chosen to be "topologically" close to the final family. One simple way to choose the initial family is to choose a family that-upon using the original CGM method-converges locally to one of the families of invariant tori, and then inclusion of sufficient resonant and fast terms will insure that this family will converge globally into an IRR basis.

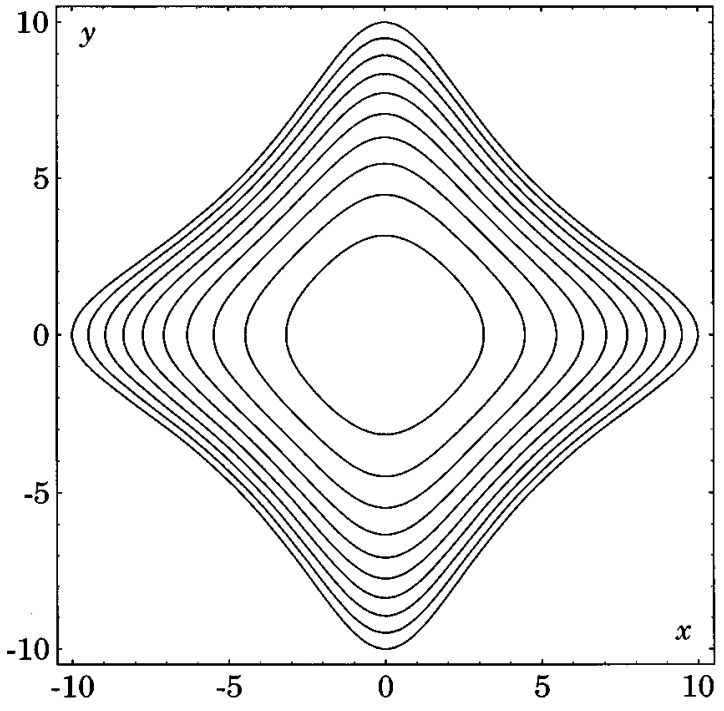

FIG. 1. Contours at $E=5,10, \ldots, 50$ of the Pullen-Edmonds potential.

\section{A NUMERICAL EXAMPLE}

In this section we illustrate our method with the PullenEdmonds Hamiltonian, ${ }^{27}$

$$
H=\frac{1}{2}\left(p_{x}^{2}+\omega_{x}^{2} x^{2}\right)+\frac{1}{2}\left(p_{y}^{2}+\omega_{y}^{2} y^{2}\right)+\epsilon x^{2} y^{2} .
$$

With the parameters $\omega_{x}=\omega_{y}=1$ and $\epsilon=0.05$ Pullen and Edmonds ${ }^{27}$ investigated the problem of the existence of regular and irregular components of the quantum spectrum. The classical dynamics and the classical/quantum mechanical correspondence between highly excited stated states has been studied by Anchell. ${ }^{28}$ The potential is totally bound with diamond-shaped contours (see Fig. 1) leading to four modes of non-linear oscillation, each corresponding to the four symmetry axis (two "local" and two "normal" modes). The choice of this Hamiltonian in our case is somewhat arbitrary but it has the quality of being simple and, yet, it exhibits some qualitative features that nicely illustrate our method. At energies lower than 20, the classical motion is quasiintegrable with the only active resonance being primary resonance between the uncoupled oscillators, whereas higher order resonances and fast terms become active at higher energies which ultimately makes the classical motion chaotic. $^{27,28}$ In the quasi-integrable regime, quite strong dynamical tunneling exists between the "local" modes. These features imply that we can illustrate our method in the case where only a single resonance condition has to be taken into account (leading to a block-diagonal Hamiltonian) and the case where more terms need be included. Furthermore, the simplicity of the Hamiltonian makes low order perturbation theory very easy and the results obtained this way can then easily be compared with our results. All numerical calculations as well as symbolic manipulations presented in this section were performed using MATHEMATICA.

Introducing the action-angle variables of the uncoupled oscillators (representing the zeroth order "local" modes), 


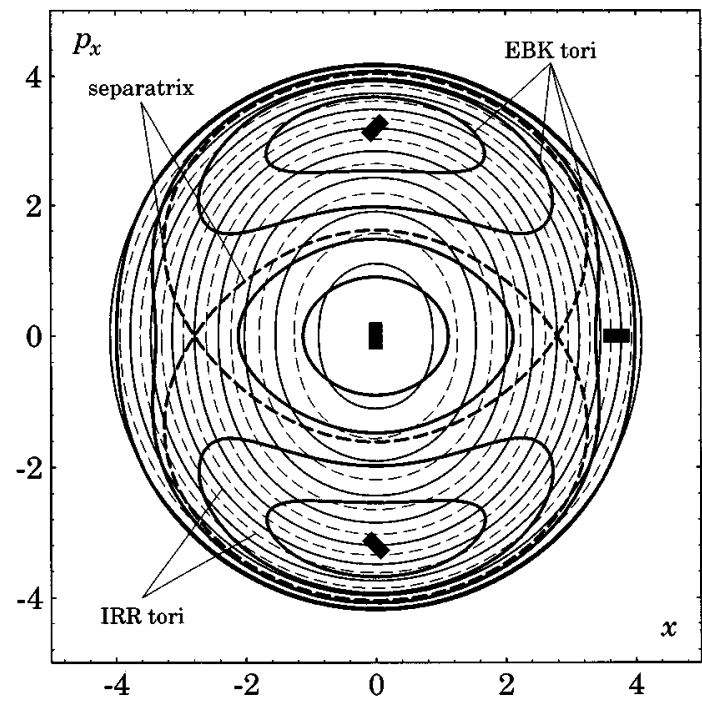

FIG. 2. Surface of section at $I_{1}^{f}+I_{2}^{f}=9$ (the sum of the good actions of the particular torus) of the tori defining an intrinsic resonance representation.

$$
\begin{aligned}
& x=\left(\frac{2 J_{1}}{\omega_{x}}\right)^{1 / 2} \cos \theta_{1}, \quad p_{x}=-\left(2 J_{1} \omega_{x}\right)^{1 / 2} \sin \theta_{1}, \\
& y=\left(\frac{2 J_{2}}{\omega_{y}}\right)^{1 / 2} \cos \theta_{2}, \quad p_{y}=-\left(2 J_{2} \omega_{y}\right)^{1 / 2} \sin \theta_{2},
\end{aligned}
$$

the Hamiltonian reads as

$$
\begin{aligned}
H(\mathbf{J}, \boldsymbol{\theta})= & \omega_{x} J_{1}+\omega_{y} J_{2}+\epsilon \frac{J_{1} J_{2}}{\omega_{x} \omega_{y}}\left\{1+\cos 2 \theta_{1}+\cos 2 \theta_{2}\right. \\
& \left.+\frac{1}{2}\left(\cos 2\left(\theta_{1}-\theta_{2}\right)+\cos 2\left(\theta_{1}+\theta_{2}\right)\right)\right\} .
\end{aligned}
$$

Choosing the same parameters as Pullen and Edmonds, let us first consider a low-energy situation (energy around $E=9$ was chosen in this case) where the system is quasiintegrable. The nine-fold degeneracy of the resonant harmonic oscillator at $E=9$ is broken by the non-linear couplings.

The invariant tori, which fill up, practically, all phase space, are separated into two families ("local" and "normal" modes) each defined by its own set of good actions. A straightforward application of the CGM method around $E=9$ produces for $\hbar=1$ eight EBK quantized invariant tori satisfying the condition $I_{1}^{f}+I_{2}^{f}=9$ (where the superscript indicates the two different definitions of actions); four obtained with the action-angle variables defined in Eq. (23) as starting point and four obtained starting with action-angle variables rotated $\pi / 4$ in configuration space with respect to these. A surface of section at $y=0, p_{y}>0$, of these eight tori is shown in Fig. 2, where the EBK tori are drawn with thick curves. The inner and outer island correspond to the "local" modes whereas the two intervening islands are the "normal" modes (the small rectangle in each island gives an idea of the way the tori of that island look like in configuration space). Interpolation yields the EBK-quantized separatrix between the islands (shown with thick dashed curves in Fig. 2). The nine
TABLE I. Quantum eigenenergies in the quasi-integrable regime and their semi-classical estimates obtained via different methods.

\begin{tabular}{crrrr}
\hline \hline State & \multicolumn{1}{c}{ QM } & \multicolumn{1}{c}{ EBK } & \multicolumn{1}{c}{ BG(4) } & \multicolumn{1}{c}{ IRR } \\
\hline 45 & 10.0133 & 10.0045 & 10.1985 & 10.0169 \\
44 & 10.0129 & 10.0045 & 10.1985 & 10.0162 \\
43 & 9.6987 & 9.6835 & 9.8519 & 9.6988 \\
42 & 9.6859 & 9.6835 & 9.8435 & 9.6876 \\
41 & 9.5166 & 9.4773 & 9.5905 & 9.5150 \\
40 & 9.4265 & 9.4041 & 9.5045 & 9.4219 \\
39 & 9.3806 & 9.4041 & 9.4199 & 9.3747 \\
38 & 9.1577 & 9.1576 & 9.1819 & 9.1514 \\
37 & 9.1548 & 9.1576 & 9.1731 & 9.1482 \\
\hline \hline
\end{tabular}

EBK energies obtained in this way are presented in the third column of Table I. The second column shows the quantum eigenenergies in the corresponding energy range obtained by diagonalization in the harmonic oscillator basis. The eight EBK energies of the quantized invariant tori are pair-wise degenerate. The degeneracy is broken in the quantum eigenenergies, indicating the existence of dynamical tunneling.

Now, let us turn to a low order perturbation treatment. For the Pullen-Edmonds Hamiltonian, the Hamiltonian obtained by first order perturbation theory ${ }^{20}$ and the fourth order Birkhoff-Gustavson normal form ${ }^{22}$ coincide, and in the case of no low order resonance $\left(\omega_{x} / \omega_{y} \neq \frac{1}{2}, 2,1\right)$ the result is

$$
\widetilde{H}(\mathbf{I}, \boldsymbol{\phi})=\omega_{x} I_{1}+\omega_{y} I_{2}+\epsilon \frac{I_{1} I_{2}}{\omega_{x} \omega_{y}},
$$

and in the case of a $1: 1$ resonance $\left(\omega_{x}=\omega_{y}=1\right)$ one obtains

$$
\widetilde{H}(\mathbf{I}, \boldsymbol{\phi})=I_{1}+I_{2}+\epsilon I_{1} I_{2}\left(1+\frac{1}{2} \cos 2\left(\phi_{1}-\phi_{2}\right)\right) .
$$

In the latter case, the semi-classical Hamiltonian matrix becomes (for $\hbar=1$ )

$$
\begin{aligned}
H_{n, m}= & \left\{\left(n_{1}+1 / 2\right)+\left(n_{2}+1 / 2\right)+\epsilon\left(n_{1}+1 / 2\right)\left(n_{2}+1 / 2\right)\right\} \\
& \times \delta_{n, m}+\frac{1}{2} \epsilon\left(n_{1}-\frac{1}{2}\right)\left(n_{2}+\frac{3}{2}\right) \delta_{n, m+2 r} \\
& +\frac{1}{2} \epsilon\left(n_{1}+\frac{3}{2}\right)\left(n_{2}-\frac{1}{2}\right) \delta_{n, m-2 r}
\end{aligned}
$$

where $\mathbf{r}=(1,-1)$. This is obviously a block diagonal matrix, where each block is characterized by the conserved quantity $I_{1}+I_{2}=n_{1}+n_{2}+1$. Diagonalizing the block characterized by $I_{1}+I_{2}=9$ gives the results listed in the fourth column of Table I. Although the energies are no longer pair-wise degenerate, the results are considerably less accurate than the EBK energies. Therefore, a perturbation treatment must certainly be carried to a much higher order for this system to give useful results.

Alternatively, we now apply the IRR method. The starting point is the Hamiltonian in the zeroth order action-angle variables, Eq. (24). We then search iteratively for solutions (I, $\boldsymbol{\phi})$ of the modified Hamilton-Jacobi equation, Eq. (16), with $\mathbf{r}=(1,-1)$ for all integer and half-integer actions $I_{1}$ and 


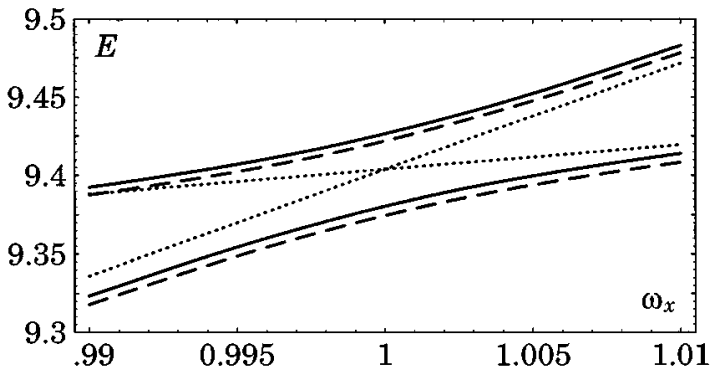

FIG. 3. Crossing of EBK energies and anti-crossing of quantum and IRR eigenenergies in the quasi-integrable regime as a function of the frequency ratio of the unperturbed system.

$I_{2}$ satisfying $I_{1}+I_{2}=9$. All the 17 tori defined by this condition can be found and these tori are also shown in Fig. 2 with thin continuous curves (integer quantization) and thin dashed curves (half-integer quantization). Note that these curves are oblivious to the 1:1 resonance local-normode structures present in the surface of section. This is actually what we desire and is a result of using the corresponding resonance term in Eq. (16) on the right hand side. The main point is that the tori of the modified problem, Eq. (16), nicely interpolate between resonance islands and make an optimal basis for describing the true eigenstates. The diagonalization of the $9 \times 9$ semi-classical block obtained this way gives the results in the fourth column of Table I. These energies are in very good agreement with the quantum eigenenergies. This last step is a "uniform" one, i.e. diagonalizing a small basis. All the methods discussed here have been uniform (i.e., incorporating quantum solutions) in some sense. For example in reduction to a pendulum Hamiltonian via a canonical transformation (ignoring fast terms) one uses finally the pendulum eigenstates, not the semi-classical states, which would again be degenerate at the simplest level. (We do not consider a final step of a tunnel integral rather than a uniform approximation; this is a possibility if the barriers are robust and tunneling is small. The small diagonalization is much more general and handles weak barriers, etc.) Integer tori couple diagonal elements separated by an odd number of quanta (as for instance two neighbors) and half-integer tori couple diagonal elements separated by an even number of quanta.

Furthermore, we have considered the result of solving Eq. (16) in a slightly non-resonant case. Figure 3 shows the anti-crossing of the two quantum eigenenergies of states 39 and 40 (continuous curves) as the parameter $\omega_{x}$ is swept around the resonant value $\omega_{y}=1$. The crossing of the corresponding EBK energies is also shown (dotted curves). The IRR eigenvalues (dashed curves) anti-cross and approximate fairly well the quantum ones throughout the anti-crossing region.

We turn to higher energy where the dynamics is chaotic. In this case the inclusion of only the resonance vector is not sufficient to obtain convergence; a second vector has to be included. Inclusion of the fast terms arising from the vector $\mathbf{s}=(1,1)$ is sufficient to obtain convergence at energies around 50. Of course, the Hamiltonian is no longer block

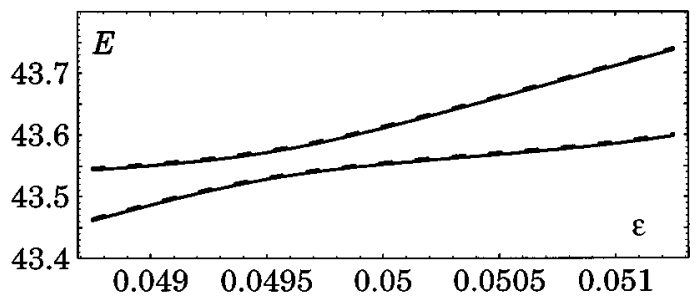

FIG. 4. Quantum and IRR eigenenergies in the chaotic regime as a function of the perturbation strength.

diagonal but the coupling structure is still simplified with the blocks yielded by $\mathbf{r}=(1,-1)$ coupled by the increasingly strong matrix elements generated by $\mathbf{s}$. Varying the coupling strength $\epsilon$, Fig. 4 shows the anti-crossing of two quantum energies in the classically chaotic regime (continuous curves). The corresponding eigenvalues of the IRR are shown with the dashed curves illustrating an excellent agreement.

\section{CONCLUSIONS}

The structure of a matrix representation of a Hamiltonian depends of course on the basis in which the matrix elements are evaluated. In the present work we have been concerned with a system of coupled oscillators. In a semi-classical representation, the basis essentially lives on tori in phase space given by the choice of action-angle variables. A natural choice for a quantum mechanical basis would be the eigenfunctions of the uncoupled system, and correspondingly one choice for the semi-classical basis is the tori of the uncoupled system. This choice may not however be the most convenient or optimal. The coupling structure in a semi-classical representation is determined by the angular dependence of the classical Hamiltonian in action-angle variables defining that representation. The goal of finding an optimal representation is thus to find a basis in which the angular dependence of the classical Hamiltonian is minimal.

In previous works the "optimal" action-angle variables have been obtained by classical perturbation theory. In this paper we have presented an alternative, non-linear iterative procedure. In the perturbative approaches one does not work directly with the dynamics of the classical system but merely performs a series of canonical transformations on the Hamiltonian itself. The input in both cases is the desired angular dependence in the final action-angle variables. In rigorous perturbation theory these only correspond to exact resonances in the zeroth order Hamiltonian and other terms must be put in by hand. Since the true dynamics is not considered there is, in general, no knowledge about how well the Hamiltonian in the final action-angle variables approximate the original Hamiltonian. On the other hand, in our approach we search for actual tori. The existence of these tori ensures that anticipated angular dependence is sufficient, whereas if the tori can not be found, the input is insufficient. Thus, our method takes into account the true dynamics of the classical system incorporating terms corresponding to the "active" resonances and, perhaps at high energy, their corresponding 
fast terms. A representation constructed on such a resonant basis we termed an intrinsic resonance representation (IRR). The main point is that our basis is small, and physically motivated (incorporating good quantum numbers for example).

The computational determination of such a basis is based on a generalization of the Chapman-Garret-Miller (CGM) method originally designed to finding invariant tori, where they actually exist, by solving the Hamilton-Jacobi equation iteratively. The generalization, embodied in Eq. (16), incorporates an inhomogeneous term in the Chapman-GarretMiller (CGM) method, modifying the Hamilton-Jacobi equation. Again a solution may or may not exist and if not further angular terms can be tried. This should be contrasted to the determination of invariant tori by perturbation theory where the actual existence is not taken into account.

In the context of semi-classical quantization the IRR tori should exist for integer and half-integer actions. The greatest advantage of an IRR basis arises when the number of necessary resonance and "fast" terms is smaller than the number of degrees of freedom since this implies the existence of global invariants of the motion and these are built into the basis, incorporating good quantum numbers. We have illustrated our method on a two-dimensional system of coupled oscillators. With the parameters chosen this system posses a primary resonance at all energies. At low energies this is the only "active" resonance and the semi-classical Hamiltonian is in block form. The results obtained by diagonalizing such a block are in very good agreement with the quantum mechanical eigenenergies reproducing anti-crossings due to dynamical tunneling. We have also performed a low order perturbation calculation which shows that for this simple system with a relatively small interaction term, low order perturbation theory is insufficient, justifying the need for a more accurate method. At lower energies a single "inhomogeneous" angular dependence is required in the generalized Hamilton-Jacobi approach, but at higher energies a second term is required. The Hamiltonian is then no longer in block form. However the physical content of the coupling terms is more transparent since they are due to a more complicated classical dynamics of the system. The semi-classical eigenenergies are in excellent agreement with the quantum ones also at higher energies.

\section{ACKNOWLEDGMENTS}

This work was partly supported by the Danish Natural Research Foundation, and the National Science Foundation under Grant No. NSF CHE-9321260. The support of ITAMP at the Harvard-Smithsonian Center for Astrophysics is also acknowledged.

${ }^{1}$ W. R. Greenberg, A. Klein, and C.-T. Li, Phys. Rev. Lett. 75, 1244 (1995).

${ }^{2}$ I. C. Percival and N. Pomphrey, Mol. Phys. 31, 97 (1976).

${ }^{3}$ S. Chapman, B. C. Garrett, and W. H. Miller, J. Chem. Phys. 64, 502 (1976).

${ }^{4}$ C. Jaffé and W. P. Reinhardt, J. Chem. Phys. 71, 1862 (1979).

${ }^{5}$ K. S. Sorbie and N. C. Handy, Mol. Phys. 32, 1327 (1976); 33, 1319 (1977); C. W. Eaker, G. C. Schatz, N. De Leon, and E. J. Heller, J. Chem. Phys. 81, 5913 (1984).

${ }^{6}$ C. C. Martens and G. S. Ezra, J. Chem. Phys. 86, 279 (1987).

${ }^{7}$ M. S. Child, Semiclassical Mechanics With Molecular Applications (Oxford University Press, Oxford, 1991).

${ }^{8}$ R. T. Swimm and J. B. Delos, J. Chem. Phys. 71, 1706 (1979).

${ }^{9}$ C. Jaffé and W. P. Reinhardt, J. Chem. Phys. 77, 5191 (1982); R. B. Shirts and W. P. Reinhardt, ibid. 77, 5204 (1982).

${ }^{10}$ C. Jaffé and P. Brumer, J. Chem. Phys. 73, 5646 (1980).

${ }^{11}$ M. J. Davis and E. J. Heller, J. Chem. Phys 75, 246 (1981).

${ }^{12}$ E. J. Heller, J. Phys. Chem. 99, 2625 (1995).

${ }^{13}$ S. Takada, J. Chem. Phys. 104, 3742 (1996).

${ }^{14}$ T. Uzer, D. W. Noid, and R. A. Marcus, J. Chem. Phys. 79, 4412 (1983); T. Uzer and R. A. Marcus, J. Chem. Phys. 81, 5013 (1984).

${ }^{15}$ D. Farrelly and T. Uzer, J. Chem. Phys. 85, 308 (1986).

${ }^{16}$ C. Jaffé and P. Brumer, J. Chem. Phys. 82, 2330 (1985); C. Jaffé, ibid. 88 , 7603 (1988)

${ }^{17}$ M. E. Kellman and E. D. Lynch, J. Chem. Phys. 85, 5855 (1986).

${ }^{18}$ L. E. Fried and G. S. Ezra, J. Chem. Phys 86, 6270 (1987).

${ }^{19}$ E. L. Silbert, III, J. Chem. Phys 88, 4378 (1988).

${ }^{20}$ A. J. Lichtenberg and M. A. Lieberman, Regular and Chaotic Dynamics, 2nd ed. (Springer-Verlag, New York, 1992).

${ }^{21}$ F. G. Gustavson, Astron. J. 71, 670 (1966).

${ }^{22}$ M. Robnik, J. Phys. A Math. Gen. 17, 109 (1984).

${ }^{23}$ M. E. Kellman, J. Chem. Phys. 93, 6630 (1990).

${ }^{24}$ W. P. Reinhardt, J. Phys. Chem. 86, 2158 (1982).

${ }^{25}$ R. L. Warnock and R. D. Ruth, Physica 26D, 1 (1987).

${ }^{26}$ Kaasalainen and J. J. Binney, Phys. Rev. Lett. 73, 2377 (1994); Mon. Not. R. Astron. Soc. 268, 1033, 1041 (1994).

${ }^{27}$ R. A. Pullen and A. R. Edmonds, J. Phys. A Math. Gen. 14, L477 (1981).

${ }^{28}$ J. L. Anchell, J. Chem. Phys. 92, 4342 (1990). 